\title{
Estimates of an Eigen Value of Some Isoperimetric Inequalities
}

\author{
Osman Abdallah A. OS ${ }^{1}$, Mohammed Nour A. Rabih'2, Muntasir Suhail D. $\mathrm{S}^{3}$ \\ ${ }^{1}$ Department of Mathematics-College of Science \& Arts, Uglat Asugour - Qassim University - Saudi Arabia \\ ${ }^{2,3}$ Department of Mathematics-College of Science \& Arts, Uglat Asugour - Qassim University - Saudi Arabia \\ Department of Mathematics-College of Science-University of Bakht Er-ruda- Eddwaim -Sudan \\ ${ }^{1}$ Email: o.osman@qu.edu.sa
}

\begin{abstract}
In this paper we give an overview of results about two types of an isoperimetric Inequalities on its eigenvalues and the eigenvalues of the laplacian. We estimate the isoperimetric type constant $\Phi\left(M_{m}\right)$ of 2-dimentional Riemannian manifold $M_{m}$. ifD be the diameter of compact Riemannian manifold $M, \alpha(n)$ is the volume of the unit n-dimensional sphere, and $i(M)$ be an injectivity radius of $M$, we prove that $\frac{V o(M)}{D}>\frac{\alpha(2)^{3}}{54 \alpha(3)^{2}} i(M)^{2}$.
\end{abstract}

Keywords: Riemannian manifold, eigenvalues, compact Riemannian manifold, Laplacian

\section{Introduction}

In this paper we consider sharp isoperimetric inequalities

$$
\begin{gathered}
\frac{\operatorname{Vol}(\partial M)}{\operatorname{Vol}(M)} \geqq \frac{2 \pi \alpha(n-1)}{\alpha(n) . \mathrm{D}} \widetilde{\omega}, \\
\frac{\operatorname{Vol}(\partial M)^{n}}{\operatorname{Vol}(M)^{n-1}} \geqq \frac{2^{n-1} \alpha(n-1)^{n}}{\alpha(n)^{n-1}} \widetilde{\omega}^{n+1}
\end{gathered}
$$

where $M^{n}$ is a compact Riemannian manifold with boundary $\partial M$ and diameter $D$, and $\widetilde{\omega}$ is a constant depending on $M$. For a history of isoperimetric inequalities see [7].

In general the constant $\widetilde{\omega}$ is hard to compute, but in some interesting cases it can be estimated.

For example, we consider the following case. Let $N^{n}$ be a compact manifold without boundary. Define the isoperimetric type constants

$$
\begin{gathered}
I(N)=\inf _{s} \frac{\operatorname{Vol}(\mathbb{S})}{\min \left\{\operatorname{Vol}\left(M_{1}\right), \operatorname{Vol}\left(M_{2}\right)\right\}^{\prime}} \\
\Phi(N)=\inf _{s} \frac{[\operatorname{Vol}(\mathbb{S})]^{n}}{\left[\min \left\{\operatorname{Vol}\left(M_{1}\right), \operatorname{Vol}\left(M_{2}\right)\right\}\right]^{n-1}}
\end{gathered}
$$

where $\mathbb{S}$ runs over codimension one submanifolds of $N$ which divide $N$ into two pieces $M_{1}$, and $M_{2}$.

[4], shows that the first eigenvalue of the Laplacian of $N, \lambda_{1}(N)$, can be bounded below in terms of $I(N)$. [9], shows that $I(N)$ [and hence $\lambda_{1}(N)$ ] can be bounded below by the diameter, volume, and Ricci curvature of $N$. In this section we reproduce Yau's result, with a slightly better constant, and show that in the two dimensional case $I(N)$ can be bounded below by the volume and injectivity radius of $N$.

In [6] Peter Li ,uses $\Phi(N)$ to get lower bounds for the higher eigenvalues of the Laplacian,for forms as well as functions, and upper bounds on their multiplicities. We show that $\Phi(N)$ can also be bounded below by the volume, diameter, and Ricci curvatureof $N$, while in the two dimensional case it can be bounded by the volume and injectivity radius of $N$.
Another case where one can estimate $\widetilde{\omega}$ is where $M$ is contained in a compact manifold $N$ without boundary, and the diameter of $M$ is less than the injectivity radius of $N$. In this case $\widetilde{\omega}=1$, so the isoperimetric inequality (2) is in terms only of the dimension of $M$. As a consequence we show that the volume of a metric ball of radius $r$ in $N$, where $r$ is less than or equal to one half the injectivity radius of $N$, is bounded below by a constant times $r^{n}$, where the constant depends only on the dimension of $N$.

We next turn our attention to universal upper and lower bounds on the first eigenvalue, $\lambda_{1}$, of the Dirichlet problem for the Laplacian.

We prove a sharp lower bound for $\lambda_{1}(M)$ where $M$ is a sufficiently nice compact manifold with boundary. In particular, if $M$ is contained in a compact manifold $N$ without boundary, and the diameter $D$ of $M$ is less than the injectivity radius of $N$, then $\lambda_{1}(M) \geqq \lambda_{1}\left(\mathbb{S}_{D}^{+}\right)$where $\mathbb{S}_{D}^{+}$is a hemisphere of the constant curvature sphere of diameter $D$. Further equality holds if and only if $M$ is isometric to $\mathbb{S}_{D}^{+}$. Cheng [10] has independently shown a universal bound for such $M$; however, his bound is not sharp.

We then show that there is a constant $\gamma(n)$ depending only on $n$ such that for every compact manifold $N^{n}$ without boundary of convexity radius $c(N)$, for every $m \in N$ and every $r<c(N)$ we have

$$
\lambda_{1}(B(m, r)) \leqq \frac{\gamma(n) \operatorname{Vol}(N)^{2}}{r^{2 n+2}},
$$

Where $B(m, r)$ is the metric ball of radius $r$ about $m$. This allows us to show

$$
\lambda_{1}(N) \leqq \frac{\gamma(n) \operatorname{Vol}(N)^{2}}{c(N)^{2 n+2}} .
$$

The proof of this result borrows much from the proof in [2]. [2] shows that there is a constant $\bar{\gamma}(n)$ depending only on the dimension $n$ of $N$ such that for every rless than the injectivety radius of $N$ there is a point $m \in N$ such that

$$
\lambda_{1}(B(m, r)) \leqq \frac{\bar{\gamma}(n) \operatorname{Vol}(N)}{r^{n+2}} .
$$




\section{International Journal of Science and Research (IJSR) \\ ISSN (Online): 2319-7064}

Index Copernicus Value (2013): 6.14 | Impact Factor (2014): 5.611

Using this we get an upper bound for $\lambda_{1}(N)$ under the assumption that $N$ admits a fixed point free involutive isometry.

Let $(M, \partial M, g)$ be a smooth compact manifold $M$ with smooth boundary $\partial M$ and Riemannian metric $g$.

Let $U M \stackrel{\pi}{\rightarrow} M$ represent the unit sphere bundle with the canonical measure. For $v \in U M$ let $\gamma_{v}$ be the geodesic with $\gamma_{v}^{\prime}(0)=v$, let $\zeta^{t}(v)$ represent the geodesic flow, i.e. $\zeta^{t}(v)=$ $\gamma_{v}^{\prime}(t)$. Let $l(v)$ be the smallest value of $t>0$ (possibly $\infty$ ) such that $\gamma_{v}(t) \in \partial M$. Note $\zeta^{t}(v)$ is defined for $t \leq l(v)$. Let $\tilde{l}(v)=\sup \left\{t \mid \gamma_{v}\right.$ minimizes up to $t$ and $\left.t \leq l(u)\right\}$.

Now let the subsets $\widetilde{U} M \subset \bar{U} \bar{M} \subset U M$ be defined by

$$
\begin{aligned}
& \bar{U} \bar{M}=\{v \in U M \mid l(-v)<\infty\}, \\
& \widetilde{U} M=\{v \in U M \mid \tilde{l}(-v)=l(-v)\} .
\end{aligned}
$$

Let $\bar{U}_{p}=\left.\pi\right|_{\bar{U} \bar{M}} ^{-1}(p)$ and $\quad \widetilde{U}_{p}=\left.\pi\right|_{\widetilde{U} M} ^{-1}(p)$. Define $\quad \bar{\omega}_{p}=$ $m\left(\bar{U}_{p}\right) / m\left(U_{p}\right) \quad$ and $\quad \widetilde{\omega}_{p}=m\left(\widetilde{U}_{p}\right) / m\left(U_{p}\right)$ where $m$ represents the canonical measure on the unit sphere. Also let $\bar{\omega}=\inf _{p \in M} \bar{\omega}_{p}, \widetilde{\omega}=\inf _{p \in M} \widetilde{\omega}_{p}$.

For $p \in \partial M$ let $N_{p}$ be the inwardly pointing unit normal vector. Let $U^{+} \partial M \rightarrow \partial M$ be thebundle of inwardly pointing unit vectors. That is

$$
U^{+} \partial M=\left\{\left.u \in U M\right|_{\partial M} \mid\left\langle u, N_{\pi(u)}\right\rangle \geqq 0\right\} .
$$

Let $U^{+} \partial M$ have the local product measure, where the measure on the fibre is the measure from the upper unit hemisphere.

We will let $\alpha(n)$ represent the volume of the unit $n$-sphere.

Proposition 1:For $(M, \partial M, g)$ we have:

$$
\begin{aligned}
& \int_{\bar{U} \bar{M}} f(v) d v=\int_{U^{+} \partial M} \int_{0}^{l(u)} f\left(\zeta^{r}(u)\right)\left\langle u, N_{\pi(u)}\right\rangle d r d u ;(3) \\
& \int_{\widetilde{U} M} f(v) d v=\int_{U^{+} \partial M} \int_{0}^{\tilde{l}(u)} f\left(\zeta^{r}(u)\right)\left\langle u, N_{\pi(u)}\right\rangle d r d u \text {. (4) }
\end{aligned}
$$

Where $f$ is any integrable function. In particular for $f \equiv 1$ we have:

$$
\begin{aligned}
& \operatorname{Vol}(\bar{U} \bar{M})=\int_{U^{+} \partial M} l(u)\left\langle u, N_{\pi(u)}\right\rangle d u \\
& \operatorname{Vol}(\widetilde{U} M)=\int_{U^{+} \partial M} \tilde{l}(u)\left\langle u, N_{\pi(u)}\right\rangle d u
\end{aligned}
$$

This formula occurs in [9],

\section{Corollary 2:}

$$
\begin{aligned}
& \frac{\operatorname{Vol}(\partial M)}{\operatorname{Vol}(M)} \geqq \frac{C_{1} \bar{\omega}}{l} ; \\
& \frac{\operatorname{Vol}(\partial M)}{\operatorname{Vol}(M)} \geqq \frac{C_{1} \widetilde{\omega}}{D},
\end{aligned}
$$

where $C_{1}=2 \pi \alpha(n-1) / \alpha(n), l=\sup _{v \in U^{+} \partial M}\{l(v)\}$ and $D$ is the diameter of $M$.

Note. The inequalities are both sharp when $M$ in the upper hemisphere of a constant curvature sphere. In this case $\bar{\omega}=\widetilde{\omega}=1$ and $l=D=$ diameter of the sphere.

Lemma 3: Let $M$ be a compact Riemannian manifold without boundary, such that the Ricci curvature is bounded below by $(n-1) K$. Then if $\mathbb{S}$ is any $n-1$ dimensional submanifold dividing $M$ into two pieces $M_{1}$ and $M_{2}$ we have

$$
\widetilde{\omega}_{i} \geqq \frac{\operatorname{Vol}\left(M_{j}\right)}{\alpha(n-1) \int_{0}^{D}(\sqrt{-1 / K} \sinh \sqrt{-K} r)^{n-1} d r}(i \neq j) .
$$

In particular if $\operatorname{Vol}\left(M_{i}\right) \leqq \operatorname{Vol}\left(M_{j}\right)$ then

$$
\widetilde{\omega}_{i} \geqq \frac{\operatorname{Vol}(M)}{2 \alpha(n-1) \int_{0}^{D}(\sqrt{-1 / K} \sinh \sqrt{-K} r)^{n-1} d r}
$$

where we use the convention that $(\sqrt{-1 / K} \sinh \sqrt{-K} r)$ is interpreted as $r$ if $K=0$ and as $\sqrt{1 / K} \sin (K r)$ if $K>0$. D represents the diameter of $M$.

Proof

$$
\begin{aligned}
& \operatorname{Vol}\left(M_{j}\right) \leqq \operatorname{Vol}\left(O_{p}\right)=\int_{-\widetilde{U}_{p}} \int_{0}^{C(u)} F(u, r) d r d u \\
& \leqq \widetilde{\omega}_{p} \alpha(n-1) \int_{0}^{D}(\sqrt{-1 / K} \sinh \sqrt{-K} r)^{n-1} d r .
\end{aligned}
$$

For the inequality $F(u, r) \leqq(\sqrt{-1 / K} \sinh \sqrt{-K} r)^{n-1}$, see [11], where

$$
-\widetilde{U}_{p}=\left\{u \in U_{p} \mid-u \in \widetilde{U}_{p}\right\}=\left\{u \in U_{p} \mid l(u)=\tilde{l}(u)\right\},
$$

And $F(u, r)$ is the volume form in normal polar coordinates.

Now from Corollary 2 we have $\operatorname{Vol}(\mathbb{S}) / \operatorname{Vol}\left(M_{i}\right) \geqq C_{1} \widetilde{\omega}_{i} / D$ (where $C_{1}$ is sharp). Thus using Lemma (1.2.4) we get ,(see Yau [6])

\section{Proposition 4:}

$$
I(M) \geqq \frac{\pi}{\alpha(n)} \frac{\operatorname{Vol}(M)}{D \cdot \int_{0}^{D}(\sqrt{-1 / K} \sinh \sqrt{-K} r)^{n-1} d r}
$$

(I $(M)$ was defined on page 1.$)$

Theorem 5: Let $M$ be a compact $n$-dimensional Riemannian manifold whose Ricci curvature is bounded below by $(n-1) K$. Thus Proposition 4 holds. Since $\lambda_{1}(M) \geqq$ $I(M)^{2} / 4$ we find a lower bound of $\lambda_{1}$ in terms of $\mathrm{D}, \operatorname{Vol}(M)$, and $K$. In some cases we are able to show that o must be 1 . For example let $M$ be a compact manifold without boundary and let $\mathbb{S}$ be an $n-1$ dimensional submanifold dividing $M$ into $M_{1}, M_{2}$ then we have:

Lemma 6: If the maximum distance in $M$ between any two points of $\mathbb{S i s}$ less than the injectivity radius of $M, i(M)$, then $\widetilde{\omega}_{i}=1$ for $i=1$ or 2 .

\section{Proof. Let $p \in \mathbb{S}$ then

$$
\mathbb{S} \subset B(p, i(M)) \equiv\{q \in M \mid d(p, q)<i(M)\} .
$$

Let $M_{i}$, be the piece of $M$ lying entirely inside $B(p, i(M))$. By continuity this choice isindependent of the choice of $p$. Now for $x \in M, d(x, p)<i(M)$ for every $p \in \mathbb{S}$, by the choice of $M_{i}$. Hence $\mathbb{S} \subset B(x, i(M))$. Let $M_{j}$. be the piece of $M$ lying in $B(x, i(M))$. By continuity $M_{j}$ is independent of $x$ and hence must be $M_{i}$. Thus every geodesic from $x$ minimizes up to $\mathbb{S}$. Hence $\widetilde{\omega}_{i}=1$.

If $M$ is a compact manifold without boundary and $r<i(M)$, let $B(x, r)=\{y \in M \mid d(x, y) \leqq r\}$ and $\mathbb{S}(x, r)=\partial B(x, r)=$ $\{y \in M \mid d(x, y)=r\}$.

Then Lemma $\mathbf{6}$ and Corollary $\mathbf{2}$ give: 


\title{
International Journal of Science and Research (IJSR) \\ ISSN (Online): 2319-7064
}

Index Copernicus Value (2013): 6.14 | Impact Factor (2014): 5.611

Corollary 7:

For $r<i(M) / 2$ :

$$
\frac{\operatorname{Vol}(\mathbb{S}(x, r))}{\operatorname{Vol}(B(x, r))} \geqq \frac{C_{1}}{2 r}=\frac{\pi \alpha(n-1)}{r \alpha(n)} .
$$

If $M$ is a two dimensional compact manifold without boundary and $\mathbb{S}$ divides $M$ into two pieces $M_{1}, M_{2}$ we can consider separately the cases where the length of $\mathbb{S} \geqq 2 i(M)$ and length of $\mathbb{S}<2 i(M)$ to get:

Corollary 8: For $M$ a compact 2-dimensional manifold

$$
I(M) \geqq \min \left\{\frac{4 i(M)}{\operatorname{Vol}(M)}, \frac{C_{1}}{i(M)}\right\} .
$$

Hence $\lambda_{1}$ can be bounded below by $i(M) \operatorname{and} \operatorname{Vol}(M)$.

Lemma 9: Let $M^{n}$ be a Riemannian manifold and $u \in U M$. Then for every $l \leqq C(u)$ (the distance to the cut locus in the direction $u$ ):

$$
\int_{x=0}^{x=l} \int_{z=0}^{z=l-x} F\left(\zeta^{x}(u), z\right) d z d x \geqq C(n) \frac{l^{n+1}}{\pi^{n+1}}
$$

Where $C(n)=\pi \alpha(n) / 2 \alpha(n-1)=\pi^{2} / C_{1}$ Further equality holds if and only if

$$
R\left(\gamma_{u}^{\prime}(t), \cdot\right) \gamma_{u}^{\prime}(t)=(\pi / l)^{2} \text { Idfor } 0 \leqq t \leqq l .
$$

Here $F(v, z)$ is the volume form in polar coordinates

$$
\text { [i. e. } \left.\int_{U_{p}} \int_{0}^{C(v)} F(v, z) d z d v=\operatorname{Vol}(M)\right] \text {, }
$$

$R$ is the curvature tensor and $\gamma_{u}$ is the geodesic determined by $u$.

This follows from a slight modification of [3].

Proposition 10: $\operatorname{For}(M, \partial M, g)$ we have

$$
\operatorname{vol}(M)^{2} \geqq C_{2} \int_{U^{+} \partial M}(\tilde{l}(v))^{n+1}\left\langle v, N_{\pi(v)}\right\rangle d v,
$$

with $C_{2}=\alpha(n) / 2 \pi^{n} \alpha(n-1)$. Equality holds for the upper hemisphere of a constant curvature sphere.

Proof.

$$
\begin{gathered}
\operatorname{Vol}(M)^{2} \geqq \int_{M} \int_{U_{p}} \int_{0}^{\tilde{l}(u)} F(u, t) d t d u d p= \\
\int_{U M} \int_{0}^{\tilde{l}(u)} F(u, t) d t d u \\
\geqq \int_{\widetilde{U} M} \int_{0}^{\tilde{l}(u)} F(u, t) d t d u(9) \\
=\int_{U^{+} \partial M} \int_{0}^{\tilde{l}(v)} \int_{0}^{\tilde{l}\left(\zeta^{\mathbb{S}}(v)\right)} F\left(\zeta^{s}(v), t\right)\left\langle v, N_{\pi(v)}\right\rangle d t d s d v \\
\geqq \int_{U^{+} \partial M}\left[\int_{0}^{\tilde{l}(v)} \int_{0}^{\tilde{l}(v)-\mathbb{S}} F\left(\zeta^{\mathbb{S}}(v), t\right) d t d s\right]\left\langle v, N_{\pi(v)}\right\rangle d v \\
\geqq \frac{C(n)}{\pi^{n+1}} \int_{U^{+} \partial M}(\tilde{l}(v))^{n+1}\left\langle v, N_{\pi(v)}\right\rangle d v \cdot(10)
\end{gathered}
$$

The above follows from Proposition 1, Lemma 9, and the fact that $\tilde{l}\left(\zeta^{\mathbb{S}}(v)\right) \geqq \tilde{l}(v)-\mathbb{S}$. Equality holds for the upper hemisphere of a sphere at each stage.

Theorem 11: $\operatorname{For}(M, \partial M, g)$ we have the isoperimetric inequality:

$$
\frac{\operatorname{vol}(\partial M)^{n}}{\operatorname{vol}(M)^{n-1}} \geqq C_{3} \widetilde{\omega}^{n+1},
$$

where $C_{3}=2^{n-1} \alpha(n-1)^{n} / \alpha(n)^{n-1}$.
Equality holds if and only if $\widetilde{\omega}=1$ and $M$ is the upper hemisphere of a constant curvature sphere.

Proof: From Proposition 10 and a Hölder inequality we have

$$
\begin{gathered}
\operatorname{vol}(M)^{2} \geqq C_{2} \int_{U^{+} \partial M}(\tilde{l}(u))^{n+1}\left\langle u, N_{\pi(u)}\right\rangle d u \\
\geqq C_{2} \frac{\left\{\int_{U^{+} \partial M} \tilde{l}(u)\left\langle u, N_{\pi(u)}\right\rangle d u\right\}^{n+1}}{\left\{\int_{U^{+} \partial M}\left\langle u, N_{\pi(u)}\right\rangle d u\right\}^{n}}, \text { (11) }
\end{gathered}
$$

using Proposition 1 we have

$$
\begin{gathered}
\operatorname{vol}(M)^{2} \cdot\left\{\int_{U^{+} \partial M}\left\langle u, N_{\pi(u)}\right\rangle d u\right\}^{n} \geqq C_{2} \operatorname{vol}(\widetilde{U} M)^{n+1} \\
\geqq C_{2}[\widetilde{\omega} \alpha(n-1) \operatorname{vol}(M)]^{n+1}
\end{gathered}
$$

giving

$$
\frac{\operatorname{Vol}(\partial M)^{n}}{\operatorname{Vol}(M)^{n-1}} \geqq C_{3} \widetilde{\omega}^{n+1} .
$$

To compute $C_{3}$ one need only note that equality holds everywhere for upper hemisphere.

To order for equality to hold we must have equality in (9), (10) and (11). Equality in(11) implies $\tilde{l}(v)$ is a constant $l$ almost everywhere in $U^{+} \partial M$. Equality in (9) implies $\widetilde{\omega}=1$. Equality in (10) implies equality in Lemma 9. Thus we see that $M$ must have constant curvature equal to $(\pi / l)^{2}$.

For $p$ an interior point of $M, x \in \mathbb{S}^{n}$, the sphere of curvature $(\pi / l)^{2}$, and $I: T_{p} M \rightarrow T_{x} \mathbb{S}^{n}$ an isometry, we see that $\operatorname{Exp}_{p_{x}} \circ I \circ \operatorname{Exp}_{p}^{-1}: M \rightarrow \mathbb{S}^{n}$ must be an isometry by [5]. To see that the image is a hemisphere one need only look at $q \in \partial M$ and note that $\tilde{l}(q)=l(q)=l$.

The equality condition only says that the upper hemisphere minimizesVol $(\partial M)^{n} / \operatorname{Vol}(M)^{n-1} \quad$ over $\quad$ spaces $(M, \partial M, g)$ with $\widetilde{\omega}=1$.

Consider $M$ a compact Riemannian manifold without boundary, and $\mathbb{S}$ a codimension one submanifold dividing $M$ into two pieces $M_{1}$ and $M_{2}$. If the maximum distance in $M$ between any two points of $\mathbb{S}$ is less than the injectivity radius, then we can combine Lemma 6 and Theorem 11 to get

$$
\frac{\operatorname{Vol}(\mathbb{S})^{n}}{\min \left\{\operatorname{Vol}\left(M_{1}\right), \operatorname{Vol}\left(M_{2}\right)\right\}^{n-1}} \geqq C_{3}=\frac{2^{n-1}}{\alpha(n)^{n-1}} .
$$

Using this in the case that $M$ is two dimensional we see:

Proposition 12: Let $M$ be a compact 2-dimensional Riemannian manifold then: $\Phi(M) \geqq 8 i(M)^{2} / \operatorname{Vol}(M)$, which is sharp for a constant curvature sphere.

Proof: Since $n=2$ we can assume that $\mathbb{S}$ is a smooth closed curve of length $l$.ifl $\geqq 2 i(M)$ then

$$
\begin{gathered}
\frac{\operatorname{Vol}(\mathbb{S})^{n}}{\min \left\{\operatorname{Vol}\left(M_{1}\right), \operatorname{Vol}\left(M_{2}\right)\right\}^{n-1}} \geqq \frac{4(i(M))^{2}}{\frac{\operatorname{Vol}(M)}{2}} \\
=\frac{8 i(M)^{2}}{\operatorname{Vol}(M)} .
\end{gathered}
$$

If $l<2 i(M)$ then by the above

$$
\begin{gathered}
\frac{\operatorname{Vol}(\mathbb{S})^{n}}{\min \left\{\operatorname{Vol}\left(M_{1}\right), \operatorname{Vol}\left(M_{2}\right)\right\}^{n-1}} \\
\geqq \frac{2(2 \pi)^{2}}{4 \pi}=2 \pi .
\end{gathered}
$$

Now in [1], and [10], show $\operatorname{Vol}(M) \geqq 4 i(M)^{2} / \pi$ Thus

\section{Volume 5 Issue 3, March 2016}

\author{
www.ijsr.net
}

Licensed Under Creative Commons Attribution CC BY 


\section{International Journal of Science and Research (IJSR)}

ISSN (Online): 2319-7064

Index Copernicus Value (2013): 6.14 | Impact Factor (2014): 5.611

$2 \pi \geqq 8 i(M)^{2} / \operatorname{Vol}(M)$.

For $n \geqq 2$ we need only combine Theorem 11 with Lemma 3 to get:

\section{Theorem 13:}

$$
\Phi(M) \geqq C_{4}\left(\frac{\operatorname{Vol}(M)}{\int_{0}^{D}(\sqrt{-1 / K} \sinh \sqrt{-K} r)^{n-1} d r}\right)^{n+1},
$$

with the same convention as Lemma 3 for $K \geqq 0 . C_{4}=$ $1 / 4 \alpha(n-1) \alpha(n)^{n-1}$.

Now Proposition 12 and Theorem 13 can be applied to the results of [6]. Thus we get a lower bound on the higher eigenvalues of $M$ as well as upper bounds on theirmultiplicities in terms of the volume of $M$, the diameter of $M$, and a lower bound on the Ricci curvature of $M$.

Note that :For $(M, \partial M, g)$ we can consider

$$
\Phi(M)=\inf _{\mathbb{S}} \frac{\operatorname{Vol}(\mathbb{S})^{n}}{\min \left\{\operatorname{Vol}\left(M_{1}\right), \operatorname{Vol}\left(M_{2}\right)\right\}^{n-1}},
$$

Where $\mathbb{S}$ moves over submanifolds dividing $M$ into two pieces $M_{1}$ and $M_{2}(\mathbb{S} \cap \partial M$ not necessarily empty). If for given $\mathbb{S}$ we let $\widetilde{U} M$ be the set of vectors whose geodesies minimize up to the point they intersect $\mathbb{S}$, and define $\widetilde{\widetilde{\omega}}$ analogously, then the same method will give an isoperimetric inequality. If $M$ is geodesically convex, then an argument similar to Lemma 3 will put a lower bound on $\widetilde{\widetilde{\omega}}$. This will give a lower bound on $\Phi(M)$.

Let $M$ be a compact Riemannian manifold without boundary. Define

$$
r_{p}(M)=
$$

$\inf \{0<r \mid(B(p, r), \mathbb{S}(p, r), g)$ has $\widetilde{\omega}>1\}$

Since $\widetilde{\omega}=1$ is equivalent to the statement that the cut locus to any interior point of $B(p, r)$ lies outside $B(p, r)$, we see that $r_{p}(M) \geqq i(M) / 2$ for all $p \in M$.

Corollary 14:Let $M_{m}$ be a compact 2-dimensional Riemannian manifold then: $\Phi\left(M_{m}\right) \geqq 8 i\left(M_{m}\right)^{2} / \operatorname{Vol}\left(M_{m}\right)$, which is sharp for a constant curvature sphere [11] .

Proof.Since $n=2$ we can assume that $\mathbb{S}$ is a smooth closed curve of length $l$.if $l \geqq 2 i\left(M_{m}\right)$ then

$$
\begin{gathered}
\frac{\operatorname{Vol}(\mathbb{S})^{n}}{\min \left\{\operatorname{Vol}\left(M_{m+1}\right), \operatorname{Vol}\left(M_{m+2}\right)\right\}^{n-1}} \\
\geqq \frac{4\left(i\left(M_{m}\right)\right)^{2}}{\frac{\operatorname{Vol}\left(M_{m}\right)}{2}} \\
=\frac{8 i\left(M_{m}\right)^{2}}{\operatorname{Vol}\left(M_{m}\right)} .
\end{gathered}
$$

If $l<2 i\left(M_{m}\right)$ then by the above

$$
\begin{gathered}
\frac{\operatorname{Vol}(\mathbb{S})^{n}}{\min \left\{\operatorname{Vol}\left(M_{m+1}\right), \operatorname{Vol}\left(M_{m+2}\right)\right\}^{n-1}} \\
\geqq \frac{2(2 \pi)^{2}}{4 \pi}=2 \pi .
\end{gathered}
$$

Shows that $\operatorname{Vol}\left(M_{m}\right) \geqq 4 i\left(M_{m}\right)^{2} / \pi$ see[1],[10] .Thus $2 \pi \geqq 8 i\left(M_{m}\right)^{2} / \operatorname{Vol}\left(M_{m}\right)$.

Proposition 15 :For $r \leqq r_{p}$ (or in particular $r \leqq i(M) / 2$ )we have

$$
\begin{gathered}
\operatorname{Vol}(B(p, r)) \geqq \frac{C_{3}}{n^{n}} r^{n}, \\
\operatorname{Vol}(\mathbb{S}(p, r)) \geqq \frac{C_{3}}{n^{n-1}} r^{n-1},
\end{gathered}
$$

in particular

$$
\operatorname{Vol}\left(B\left(p, \frac{i(M)}{2}\right)\right) \geqq \frac{\alpha(n-1)^{n}}{2 n^{n} \alpha(n)^{n-1}} i(M)^{n} .
$$

Proof.By Theorem 11 for $0<t \leqq r$ :

$$
\frac{\operatorname{Vol}(\mathbb{S}(p, t))}{\operatorname{Vol}(B(p, r))^{(n-1) / n}} \geqq C_{3}^{1 / n}
$$

integrating both sides with respect to $t$ yields

$$
n \text {.Vol }(B(p, r))^{1 / n} \geqq C_{3}^{1 / n} r .
$$

This gives the first statement. The second follows from Theorem 11 and the first statement.

This relates to a question of Berger. Berger is interested in bounding the volume of a compact manifold from below in terms of the injectivity radius. In [3], he proves that $\operatorname{Vol}(M) \geqq(1 / 2)\left(\alpha(n) / \pi^{n}\right) i(M)^{n}$.Proposition 15 can be considered as a local version of this result. One has from Proposition 15 that

$$
\operatorname{Vol}(M) \geqq \operatorname{Cat}(M) \cdot \frac{C_{3}}{2^{n} n^{n}} i(M)^{n},
$$

Where $\operatorname{Cat}(M)$ is the topological category of $M$ (i.e., the number of topological $n$-balls needed to cover $M)$. To see this one need only note that for every $x \in M, B(x, i(M))$ (open) is a topological $n$-ball, then choose $x_{1} \in M$, choose $x_{2} \in M-B\left(x_{1}, i(M)\right)$, in general choose $x_{i} \in M-$ $\bigcup_{j=1}^{i-1} B\left(x_{j}, i(M)\right)$; by the definition of $\operatorname{Cat}(M)$ we can choose at least Cat $(M)$ such $x_{i}$. Now for $j \neq i, d\left(x_{i}, x_{j}\right)>$ $i(M)$ hence $\quad B\left(x_{i}, i(M) / 2\right) \cap B\left(x_{j}, i(M) / 2\right)=\emptyset$.Hence Proposition 15 gives the result.

Proposition 15 also allows us to get good lower bounds on $\operatorname{Vol}(M)$ when $r_{p}(M)$ is large forsome $p$ even though the injectivity radius may be small. Another consequence is:

Corollary 16 : Let $M$ be a compact Riemannian manifold then

$$
\frac{\operatorname{Vol}(M)}{D}>\frac{\alpha(n-1)^{n}}{2 n^{n} \alpha(n)^{n-1}} i(M)^{n-1} .
$$

Proof. Let $I$ be the integer such that $I+1>D / i(M) \geqq I \geqq$ 1. Let $\gamma$ be a minimizing geodesic from $p$ to $q$ in $M$ of length $D$. Choose points $p=x_{0}, x_{1}, x_{2}, \ldots, x_{I}=q$ along $\gamma$ such that $d\left(x_{i}, x_{i+1}\right) \geqq i(M)$. Then the geodesic balls $B\left(x_{i}, i(M) / 2\right)$ will be disjoint and have volume $\geqq\left(\alpha(n-1)^{n} /\right.$ 2nnann-1iMn. Thus

$$
\begin{gathered}
\operatorname{Vol}(M) \geqq(I+1) \frac{\alpha(n-1)^{n} i(M)^{n}}{2 n^{n} \alpha(n)^{n-1}} \\
\geqq \frac{D}{i(M)} \frac{\alpha(n-1)^{n} i(M)^{n}}{2 n^{n} \alpha(n)^{n-1}} .
\end{gathered}
$$

Corollary 17: Let $M$ be a compact Riemannian manifold [11] then

$$
\frac{\operatorname{Vol}(M)}{D}>\frac{\alpha(2)^{3}}{54 \alpha(3)^{2}} i(M)^{2}
$$

Proof. Let $1+\in$ be the integer such that $2+\in>D / i(M) \geqq$ $1+\in$. Let $\gamma$ be a minimizing geodesic from $p$ to $q$ in $M$ of length $D$. Choose points $p=x_{0}, x_{1}, x_{2}, \ldots, x_{1+\epsilon}=q$ along $\gamma$ such that $d\left(x_{i}, x_{i+1}\right) \geqq i(M)$. Then the geodesic balls $B\left(x_{i}, i(M) / 2\right)$ will be disjoint and have volume $\geqq$ $\left(\alpha(2)^{3} / 54 \alpha(3)^{2}\right) i(M)^{3}$. Thus 


\section{International Journal of Science and Research (IJSR) \\ ISSN (Online): 2319-7064}

Index Copernicus Value (2013): 6.14 | Impact Factor (2014): 5.611

$$
\begin{gathered}
\operatorname{Vol}(M) \geqq(2+\epsilon) \frac{\alpha(2)^{3} i(M)^{3}}{54 \alpha(3)^{2}} \\
\geqq \frac{D}{i(M)} \frac{\alpha(2)^{3} i(M)^{3}}{54 \alpha(3)^{2}} .
\end{gathered}
$$

We prove the following lower bound for the first eigenvalue of the Dirichlet problem for the Laplacian.

Corollary 18: Let $N$ be a complete Riemannian manifold of injectivity radius $i(N)$. Then for every $m \in N$ and every $r \geqq i(N) / 2$ we have $\lambda_{1}(B(m, r)) \geqq \lambda_{1}\left(\mathbb{S}_{2}^{+} r\right)$, with equality holding if and only if $B(m, r)$ is isometric to $\mathbb{S}_{2 r}^{+}$. [in which case $r=i(N) / 2$ ].

Theorem 19: $\operatorname{Let}(M, \partial M, g)$ be a compact Riemannian manifold with boundary such that every geodesic ray in $M$ intersects $\partial M$. (i.e., $\bar{\omega}=1$ ). Let $l$ be the maximum length of any geodesic (from boundary point to boundary point). Then we have $\lambda_{1}(M) \geqq \lambda_{1}\left(\mathbb{S}_{l}^{+}\right)$. If further every geodesic ray minimizes distance up to the point that it intersects the boundary(i.e., $\widetilde{\omega}=1$ ), then equality holds if and only if $M$ is isometric to $\mathbb{S}_{l}^{+}$.

Proof . By the minimum principle we need only show that

$$
\frac{\int_{M}|\nabla f|^{2} d m}{\int_{M} f^{2} d m} \geqq \lambda_{1}\left(\mathbb{S}_{l}^{+}\right)
$$

for all $f$ such that $\left.f\right|_{\partial M}=0$.

We first note that

$$
|\nabla f(p)|^{2}=\frac{n}{\alpha(n-1)} \int_{U_{p}}(v f)^{2} d v,
$$

Where $v f$ represents differentiation.

Using this, Proposition 1 (with $\overline{U M}=U M$ ) and the one dimensional version:

$$
\begin{gathered}
\int_{0}^{a} f^{\prime}(t)^{2} d t \geqq \frac{\pi^{2}}{a^{2}} \int_{0}^{a} f(t)^{2} d t, \\
f(0)=0, f(a)=0,
\end{gathered}
$$

with equality if and only if $f(t)=A \sin ((\pi / a) t)$, we see

$$
\begin{gathered}
\int_{M}|\nabla f|^{2} d m=\frac{n}{\alpha(n-1)} \int_{U M}(v f)^{2} d v \\
=\frac{n}{\alpha(n-1)} \int_{U^{+} \partial M} \int_{0}^{l(u)}\left[\left(\zeta^{t}(u)\right) f\right]^{2} d t\left\langle u, N_{\pi(u)}\right\rangle d u \\
\geqq \frac{n}{\alpha(n-1)} \int_{U^{+} \partial M} \frac{\pi^{2}}{l(u)^{2}} \int_{0}^{l(u)}\left[f\left(\pi\left(\zeta^{t}(u)\right)\right)\right]^{2} d t\left\langle u, N_{\pi(u)}\right\rangle d u \\
\geqq \frac{n \pi^{2}}{\alpha(n-1) l^{2}} \int_{U^{+} \partial M} \int_{0}^{l(u)}\left[f\left(\pi\left(\zeta^{t}(u)\right)\right)\right]^{2} d t\left\langle u, N_{\pi(u)}\right\rangle d u \\
=\frac{n \pi^{2}}{\alpha(n-1) l^{2}} \int_{U M}[f(\pi(v))]^{2} d v \\
=\frac{n \pi^{2}}{l^{2}} \int_{M} f^{2} d m=\lambda_{1}\left(\mathbb{S}_{l}^{+}\right) \int_{M} f^{2} d m
\end{gathered}
$$

Now we assume that equality holds. Equality holds if and only if:

(a) $l=l(u)$ for every $u \in U^{+} \partial M$ and

(b) $f\left(\gamma_{u}(t)\right)=A(u) \sin (\pi / l) t$ for all $u \in U^{+} \partial M$, where $\gamma_{u}(t)$ represents the geodesic with initial tangent vector $u$ and $A(u)$ is a constant depending on $u$.

By scaling we may assume that $\sup (f)=1$. Let $m \in M$ be such that $f(m)=1$. Then if $\gamma$ is any geodesic through $m$ (parameterized from boundary point to boundary point), $m$ will take on the maximum value of $f$ hence $m=\gamma(l / 2)$. Thus it is not hard to see:

(i) $M$ is the metric ball of radius $l / 2$ around $m$ and

$$
\partial M=\{q \in M \mid d(m, q)=l / 2\} .
$$

(ii) $f(q)=\cos [\pi(d(p, q)) / l]$ for all $q \in M$.

(iii) $A(u)=\left\langle u, N_{\pi(u)}\right\rangle$ forall $u \in U^{+} \partial M$.

Let $u \in T_{q} \partial M, q \in \partial M$. Bycontinuity $\gamma_{u}(t)$ is defined (i.e. lies in $M)$ for $0 \leqq t \leqq l$. Since $A(u)=\left\langle u, N_{\pi(u)}\right\rangle=0$ we see that $f\left(\gamma_{u}(t)\right)=0$ for all $t \leqq l$. Hence $\gamma_{u}(t) \in \partial M$ for $0 \leqq t \leqq$ $l$. Thus $\partial M$ is totally geodesic.

For $q \in \partial M$ we let $\tilde{q}$ represent the (antipodal) point $\gamma_{N_{q}}(l) \in$ $\partial M$. We now assume (as in the statement of the Theorem) that every geodesic minimizes length up to the point it intersects $\partial M$. As $M$ is the metric ball of radius $l / 2$ around $m$ the unique point of distance $l$ from $q$ is $\widetilde{q}$. Hence if $\gamma$ is any geodesic from $q$ we have $\gamma(l)=\tilde{q}$. Hence this holds for geodesies in $\partial M$. Hence the metric on $\partial M$ is that of a Blaske structure on a sphere. Hence by [3] $\partial M$ is isometric to the constant curvature sphere $\partial \mathbb{S}_{l}^{+}$. Inparticular $\operatorname{Vol}(\partial M)=$ $\operatorname{Vol}\left(\partial \mathbb{S}_{l}^{+}\right)$. Now using the assumptions of the Theorem, the fact that $l(u)=l$, and the proof of Corollary 2 we see that

Thus

$$
\frac{\operatorname{Vol}(\partial M)}{\operatorname{Vol}(M)}=\frac{\operatorname{Vol}\left(\partial \mathbb{S}_{l}^{+}\right)}{\operatorname{Vol}\left(\mathbb{S}_{l}^{+}\right)}
$$

$$
\frac{\operatorname{Vol}(\partial M)^{n}}{\operatorname{Vol}(M)^{n-1}}=\frac{\operatorname{Vol}\left(\partial \mathbb{S}_{l}^{+}\right)^{n}}{\operatorname{Vol}\left(\mathbb{S}_{l}^{+}\right)^{n-1}}=C_{3}
$$

Now the fact that every geodesic minimizes up to $\partial M$ combined with Theorem $\mathbf{1 3}$ gives $M$ is isometric to $\mathbb{S}_{l}^{+}$.

\section{References}

[1] M.Berger, "some Relations Between Volume, Volume, Injectivity Radius and Convexity radius in Riemannian Manifolds," Differential Geometry and Relativity, Reidelpublishing CO,. Dordrecht-Boston, pp.33-42, 1976.

[2] M.Berger, "Une inegality universelle poure la premiere valeur proper du laplacien," Bull . Soc . math . Fr ., T. 107 , PP33-42, 1979.

[3] A.Besse,"Manifolds All of Whose Geodesics are closed,'Ergebnisse der Mathematik, Vol .93, Springer ,Berlin-Heidelberg-New York, 1978.

[4] J.Cheeger, "Alower Bound for the Smallest Eigenvalue of the laplacian , in Problems in Analysis,"A Symposium inHonor of S.Bochner , Princeton University Press ,Princeton, pp.195-199, 1970.

[5] J.Cheeger and D.G.Ebin, "Comparison Theorems in Riemannian Geometry," . North Holland , Amsterdam, 1975.

[6] P.Li, "on the Sobolev Constant and the p-Spectrum of a Compact Riemannian Manifold," Annscient.Ec.Norm.Sup., T.13,pp.451-467, 1980 .

[7] R. Osserman, "The Isoperimetric Inequality," Bull. Amer. Math.Soc.,Vol .87,pp.1182-1238, 1978.

[8] L.A.Santalo, "Integral Geometry and Probability," Encyclopedia of Mathematics and its Applications, Addison-Wesley , London-Amsterdam-Don Mills, Ontario-Sydney-Tokyo, 1976. 


\section{International Journal of Science and Research (IJSR) \\ ISSN (Online): 2319-7064}

Index Copernicus Value (2013): 6.14 | Impact Factor (2014): 5.611

[9] S.-T.Yau , "Isoperimetric constants and the first eigenvalue of a compact Riemannian manifold," ann.Sci. Ecole Norm.sup., pp.487-507, 1975.

[10]C. B. Croke, "some Isoperimetric Inequalities and EigenvalueEstimates, Annales scientifiques de L, E.N.S., PP.419-435, 1980.

[11] Mohammed Nour Ali, "Isoperimetric controlAnd conformal upper bounds for the eigenvalues of the Laplacian and Steklov problem," Ph.D Thesis in Mathematics, University ofBakht Er-ruda , PP. 1-285, 2014.

Volume 5 Issue 3, March 2016

www.ijsr.net

Licensed Under Creative Commons Attribution CC BY 